\title{
2.5D Numerical Simulation of Relativistic Electron Bunches (REB) Interaction with Underdense and Overdense Plasmas
}

\author{
Oleg V. Batishchev ${ }^{\dagger}$, Vjacheslav I. Karas’ ${ }^{\ddagger}$, Yurii S. Sigov ${ }^{\dagger}$, Yakov B. Fainberg ${ }^{\ddagger}$ \\ ${ }^{\dagger}$ M.V.Keldysh Institute of Applied Mathematics \\ Russian Academy of Sciences \\ 125047 Miusskaya Sq., Moscow, Russia \\ ${ }^{\ddagger}$ Kharkov Institute of Physics and Technology \\ 310108, Kharkov, Ukraine
}

\begin{abstract}
The results of REB - plasma interaction numerical simulation by means of our relativistic particle EM code are presented. Nonlinear evolution of a single REB injected into underdense and overdense plasmas is studied in detail for cylindrical geometry. It is found that in case of comparable bunch and plasma densities an instantaneous amplitude of excited longitudinal electric field substantially depends on density ratio, relativistic factor and space density distribution of REB. Transversal electric field can considerably exceed the longitudinal one, causing more severe restrictions to initial phase and radial position of particles to be accelerated.
\end{abstract}

\section{INTRODUCTION}

The accelerating of charged particles by means of spacecharge waves in plasmas and in unneutralized chargeparticle beams [1] is a basic direction in collective acceleration methods. One of the main advantages of this method is that it can produce strong electric fields in plasma which are directed along the propagation direction of linear or nonlinear waves with phase velocities $v_{p h} \leq c, c$ - light velocity. This makes possible to keep continuously the wave matched with particles to be accelerated. The possibility of strong accelerating fields achievement simply follows from the circumstance that the field amplitude is proportional to the variable component of the charge density. The last can reach the equilibrium value of the plasma density. Since this density can be made very high, the electric field can reach values up to $10^{7}-10^{9} \mathrm{~V} / \mathrm{cm}[1,2]$.

Chen et al. [3] suggested a modification of Fainberg proposed acceleration method [1]: switching to acceleration by waves train excited by a finite sequence of periodically spaced REB moving through a dense plasma. The serious difficulty of plasma wake-field acceleration (PWFA) method has been eliminated by Katsouleas [4], who presented a proof that if a bunch with a uniform density distribution is replaced by one asymmetric along it's length, then due plasma wave electric field excited behind a bunch $E_{a c}$, accelerating particles injected from the outside of the system, can be many times greater than the electric field which stops the bunch $E_{s t}$. The excitation of nonlinear stationary waves in a plasma by a sequence of periodically spaced electron bunches was studied in ref. $[5,6]$, where it was shown that the electric field of the wave increases with increasing relativistic factor $\gamma$, but only in the case of comparable bunch $n_{b}$ and plasma $n_{e}$ densitics.

The experiments performed in the nonlinear regime of the PWFA displayed interesting three dimensional effects, but only one dimensional treatments of nonlinear plasma wake-fields currently exist (see for example [6] and cited references). In ref. [7] a high-intensity rigid electron beam is used to excite extremely nonlinear, transverse motion dominated plasma oscillations.

\section{SIMULATION RESULTS}

In this work the results of full-scale numerical simulation of a single or a sequence of relativistic clectron bunches in plasmas is presented. Excitation of wake fields by them is studied using our 2.5D axial-symmetric electromagnetic code [8]. Recently this code was applied to an induction accelerator [9] and modulated REB simulations [10].

\section{A. Mathematical model}

Dynamics of REB is described by relativistic Vlasov (Beliaev-Budker) equations for distribution functions of plasma species and Maxwell equations for self-consistent $E$ and $B$ fields. Two component bulk plasma $\left(m_{i} / m_{e}=\right.$ 1840 ) with density $n_{p}$ was initially cold and filled the whole simulation region $[0, Z] \times[0, R]$. Usually $Z$ and $R$ were taken as 25 and $3 c / \omega_{p}$ respectively, $\omega_{e}$ is Langmuir plasmas frequency. At the plane $Z=0$ we organized one or a train of cold relativistic electron bunches injection in accordance with a formula ( $n$ means the injected bunch number)

$n_{b} \theta\left(R_{0}-r\right) \theta\left(v_{b} t-z+(n-1) \lambda_{p}\right) \theta\left(z-v_{b} t+Z_{0}+(n-1) \lambda_{p}\right)$ Here beam velocity $v_{b}=c\left(1-1 / \gamma^{2}\right)^{1 / 2}, Z_{0}$ and $R_{0}$ initial sizes of bunches were equal to $1 / 3$ and $1 / 6 \mathrm{c} / \omega_{p}$. $\lambda_{p}=2 \pi c / \omega_{e}, n_{b}=1 / 2 n_{e^{-}}$mean density of REB. Note, that as in experiments [6], initial transversal $R_{0}$ and longitudinal $Z_{0}$ bunches sizes were less than $c / \omega_{e}$. Bunches could leave the simulation region at it's two corners $z=0$ 
and $z=Z$ as well as plasma species, which were additionally pumped back into the volume. At the inner surface of cylinder $r=R$ all particles were reflected. Boundary conditions for fields were the following: metallic at the $r=R$ and free radiation of $E M$ waves at corners. In computations we used explicit schemes, time and space steps were constant and equal to $0.05 \mathrm{c} / \omega_{p}$ and $0.025 \omega_{p}^{-1}$ respectively. So, the mesh size was $512 \times 64$, a silent start technique was used. Model particles weight was a function of their radial position, to represent plasma with a less number of particles per cell in less disturbed regions far from axis. The total number of particles was approximately 300000 . Note, that all simulations were carried out using $P C / 386 / 20$ and we applied the fast modification of particle-in-cell method.

\section{RESULTS AND DISCUSSION}

The numerical simulation of one bunch propagation in plasma demonstrates that it's transversal size changes in wide range. This leads to essential changes in it's density (up to one order) as well as in the wake field excited by them. Longitudinal distributions of transversal $E_{\boldsymbol{r}}$ and longitudinal $E_{z}$ electric fields corresponding to radius $r=R_{0}$ and time $t=18 \omega_{e}^{-1}$ are shown at Fig.1 and Fig.2 respectively (in $m_{e} c \omega_{p} / e$ units). You can see that immediately after the REB with $\gamma=430$ shapes of radial and longitudinal electric fields differ from sinusoidal, and their amplitudes are not more constant. This is due to transversal oscillations of bunch particles in self-consistent fields connected with an absence of both charge and current compensations. To study a relationship between an excited fields and a number of injected into plasma bunches with initial energy $m c^{2} \gamma$ two runs were considered.

\section{A. Comparison of two runs}

Run 1 is shown at Fig. $3\left(t=15 \omega_{e}^{-1}\right)$ and has the following parameters: $N=3, \gamma=430$. Run 2 (shown at Fig. 6 $\left.\left(t=50 \omega_{e}^{-1}\right)\right)$ has eight bunches (three ones are shown at a moment) with $\gamma=43$.

At Figs.4,7 longitudinal distributions of radial electric field $E_{r}$ and at Figs.5,8 axial electric field $E_{z}$ corresponding to Runs 1 and 2 respectively are represented. In addition, the shape of each bunch is shown in magnified scale at top of Figs.3,6. You can see that amplitudes of both longitudinal and transversal fields increase with the bunch injection. This amplitudes nevertheless are not proportional to the number of injected bunches, as it should be for "rigid" bunches. At Fig. 9 longitudinal electric field energy time dependence for Run 2 is shown. Each step on the curve corresponds to a moment, when new bunch interacts the simulated system boundary. The non-monotonic character of excited field grow take place after third bunch injection, signifying formation of non-linear regine of train propagation.

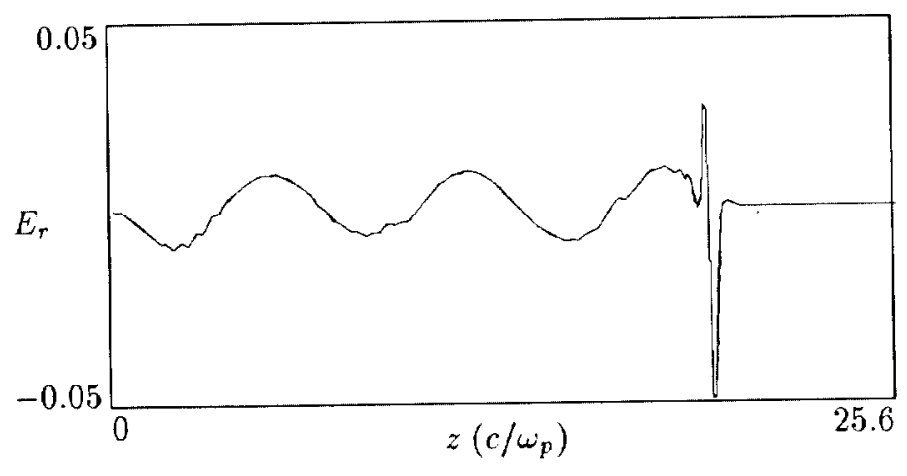

Figure 1: Transversal electric field $E_{r}$

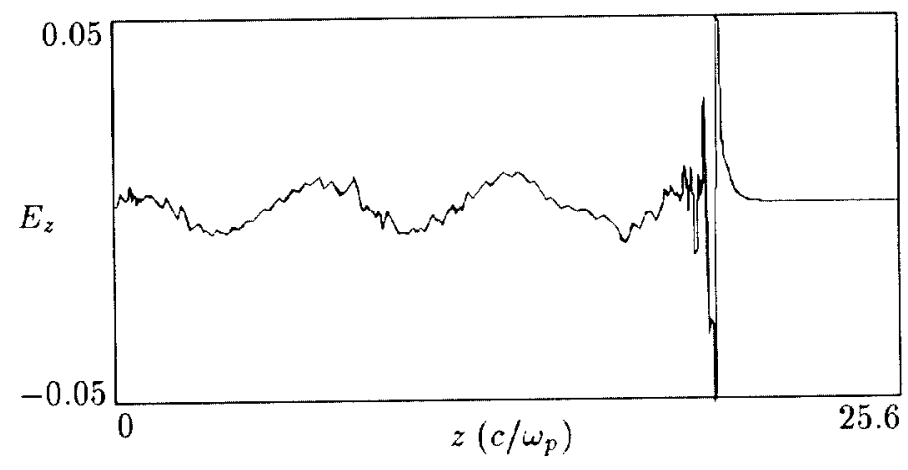

Figure 2: Longitudinal electric field $E_{z}$

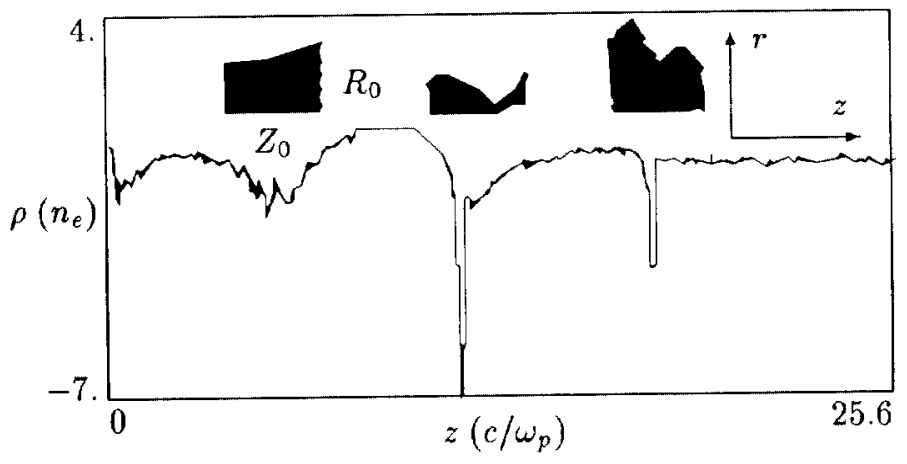

Figure 3: Three bunches, density $\rho$

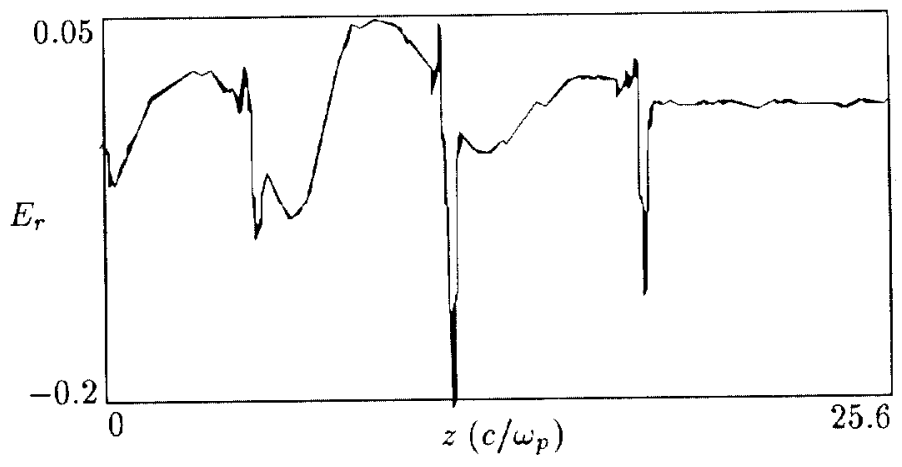

Figure 4: Three bunches, field $E_{r}$ 


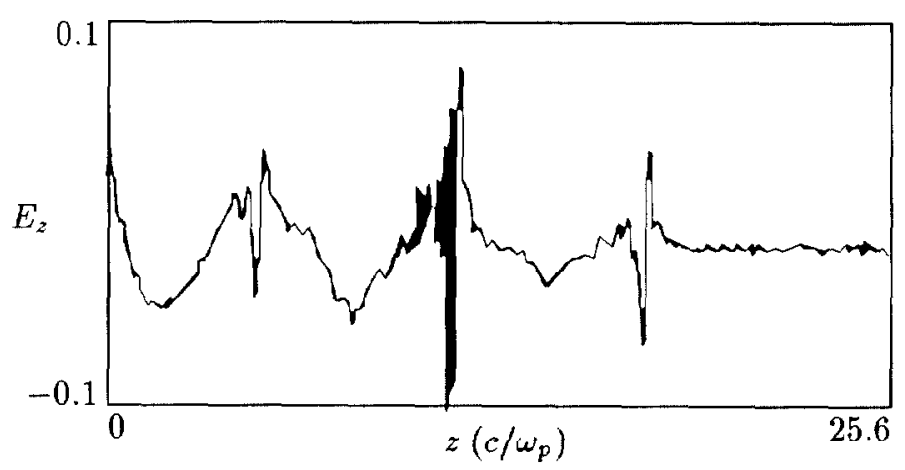

Figure 5: Three bunches, field $E_{z}$

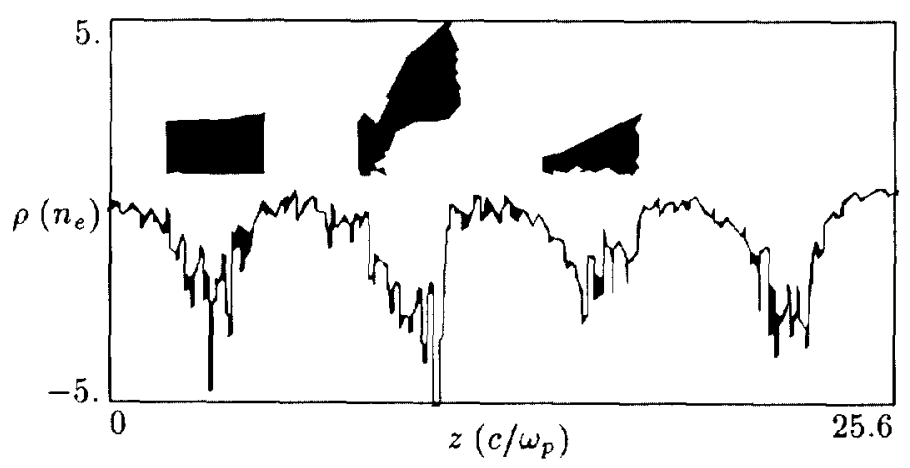

Figure 6: Eight bunches, density $\rho$

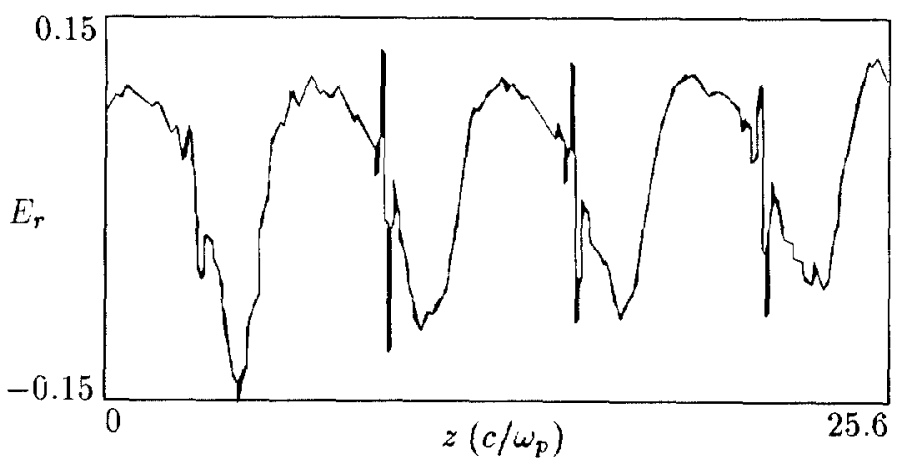

Figure 7: Eight bunches, field $E_{r}$

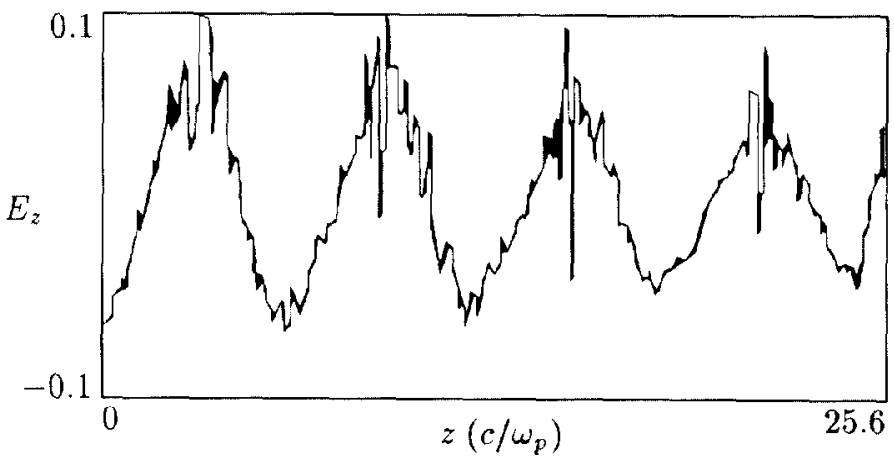

Figure 8: Eight bunches, field $E_{z}$

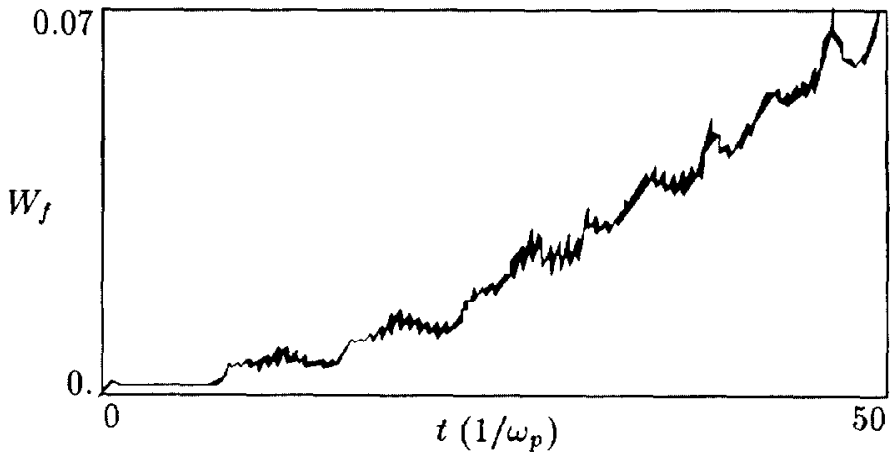

Figure 9: $E_{z}$ energy, $W_{f}(t)$

\section{REFERENCES}

[1] Ya. B. Fainberg, "The use of plasma waveguides as accelerating structures in linear accelerators", Proc. Symp. CERN, Vol. 1, 84 (1956).

[2] Ya. B. Fainberg, "Acceleration of charged particles by space-charge waves excited on plasmas by laser beams and relativistic electron beams", Sov. J. Plasma Physics, 13, No. 5, 350 (1987).

[3] P. Chen, J. M. Dawson, R. W. Huff, and T. Katsouleas, "Acceleration of electrons by the interaction of a bunched electron beam with a plasma", Phys. Rev. Lett., 54, 693 (1985).

[4] T. Katsouleas, "Physical mechanisms in the plasma wake-field accelerator", Phys. Rev. A, 33, No. 3, pp. 2056-2064 (March 1986).

[5] A. Ts. Amatuni et al., "Excitation of a high amplitude longitudinal waves in a plasma by electron bunches", Sov. J. Plasma Physics, 11, 417 (1985).

[6] J. B. Rosenzweig, "Nonlinear plasma and beam physics in plasma wake-field", Conf. 90/40 (February 1990).

FERMILAB

[7] J. B. Rosenzweig et al., "Acceleration and focusing of electrons in two dimensional nonlinear plasma wake fields", Phys. Rev. A, 44, No. 10, pp. 6189-6192 (November 1991).

[8] Discrete plasma simulation (ed. Yu. S. Sigov), Keldysh Inst. Appl. Math., Moscow (1990).

[9] V. I. Karas' et al., "Studies of space-charge neutralized ion beam induction linac for inertial confinement fusion", Particle Accelerators, 37-38, pp. 281-288 (1992).

[10] O. V. Batishchev et, al., "Self-focusing of a modulated ribbon REB in a dense plasmas", Sov. J. Plasma Physics, 19, No. 6, pp. 738-747 (1993). 